\title{
Inter-Vendor Performance of Deep Learning in Segmenting Acute Ischemic Lesions on Diffusion- Weighted Imaging: A Multicenter Study
}

Deniz Alis ( $\nabla$ drdenizalis@gmail.com )

Acıbadem University

Mert Yergin

Bahçeşehir University

Ceren Alis

Istanbul University Cerrahpaşa

Cagdas Topel

Istanbul Mehmet Akif Ersoy Research and Training Hospital

Ozan Asmakutlu

Istanbul Mehmet Akif Ersoy Research and Training Hospital

Omer Bagcilar

Istanbul Silivri State Hospital

Yeseren Senli

Istanbul University Cerrahpaşa

Ahmet Ustundag

Istanbul University Cerrahpaşa

Vefa Salt

Istanbul University Cerrahpaşa

Sebahat Dogan

Gaziosmanpaşa Taksim Eğitim ve Araştırma Hastanesi

Murat Velioglu

Fatih Sultan Mehmet Eğitim Ve Araştırma Hastanesi

Hakan Selcuk

Bakırköy Dr.Sadi Konuk Eğitim ve Araştırma Hastanesi

\section{Batuhan Kara}

Bakırköy Dr.Sadi Konuk Eğitim ve Araştırma Hastanesi

Ilkay Oksuz

Istanbul Technical University

Osman Kizilkilic

Istanbul University Cerrahpaşa

\section{Ercan Karaarslan}




\section{Research Article}

Keywords: artificial intelligence, deep learning, diffusion-weighted imaging, ischemic stroke, machine learning, magnetic resonance imaging

Posted Date: December 28th, 2020

DOl: https://doi.org/10.21203/rs.3.rs-132659/v1

License: (c) (1) This work is licensed under a Creative Commons Attribution 4.0 International License. Read Full License 


\section{Abstract}

There is little evidence on the applicability of deep learning (DL) in segmentation of acute ischemic lesions on diffusion-weighted imaging (DWI) between magnetic resonance imaging (MRI) scanners of different manufacturers. We retrospectively included DWI data of patients with acute ischemic lesions from six centers. Dataset A $(n=2986)$ and $B(n=3951)$ included data from Siemens and GE MRI scanners, respectively. The datasets were split into the training $(80 \%)$, validation $(10 \%)$, and internal test $(10 \%)$ sets and six neuroradiologist created ground-truth masks. Models $A$ and $B$ were the proposed neural networks trained on datasets $A$ and $B$ and also fine-tuned across the datasets using their validation data. Another radiologist performed the segmentation on the test sets for comparisons. The median Dice scores of models $A$ and $B$ were 0.858 and 0.857 for the internal tests, which were non-inferior to the radiologist's performance, but demonstrated lower performance than the radiologist on the external tests. Fine-tuned models $A$ and $B$ achieved median Dice scores of 0.832 and 0.846 , which were non-inferior to the radiologist's performance on the external tests. The present work shows that inter-vendor operability of deep learning for the segmentation of ischemic lesions on DWI might be enhanced via transfer learning; thereby, their clinical applicability and generalizability could be improved.

\section{Introduction}

Ischemic stroke is a significant public health problem and one of the leading causes of mortality and disability worldwide [1]. Ischemic stroke is routinely diagnosed using neuroimaging modalities, such as computed tomography (CT) and magnetic resonance imaging (MRI) [1]. Recent evidence suggests using CT over MRI due to the importance of initiating treatment early [2]. Nevertheless, MRI offers valuable information in challenging cases and for better delineation of ischemic lesions in the early stages of the disease due to its unsurpassed contrast resolution $[3,4]$. Furthermore, the volume of the ischemic core assessed using diffusion-weighted imaging (DWI) provides essential insights for decision-making and prognostication [5-8]. The current gold standard for measuring the ischemic core on DWI is manual segmentation, which is a labor-intensive, time-consuming, and tedious task; therefore, it may be omitted in daily practice due to the need for instantaneous estimation. Accordingly, several traditional machine learning- and threshold-based approaches have been proposed for segmentation of acute ischemic lesions with variable success rates $[9,10]$.

Deep learning $(\mathrm{DL})$ is a subfield of machine learning that involves using a stack of interconnected neurons for simultaneously extracting the representative features and making predictions for a given task [11]. Recent studies have demonstrated that DL, particularly convolutional neural networks (CNNs), is a robust tool for analyzing medical images for a variety of tasks, including classification, segmentation, and object detection [12]. Several studies published within the last 3 years have demonstrated the yield of deep learning in estimating the ischemic core on DWI and that DL-based methods surpassed the predecessor methods mentioned above [13-19]. However, most of the earlier efforts that utilized DL used samples obtained at a single institution and lacked independent external validation and performance comparison with a radiologist [13-17]. Despite the high-representative capacity of DL models, several 
authors have recently criticized the generalizability of DL models across datasets derived from different domains (e.g., MRI obtained with different MRI scanners or at different institutions) [20, 21].

In this study, we used a novel DL architecture, residual two-dimensional (2D) U-net with convolutional long short-term memory (ConvLSTM) unit, for automated segmentation of acute ischemic lesions on DWI using a large-scale multicenter dataset. The aims of this study were the following: first, to externally validate the performance of DL models across different MRI vendors; second, to compare the diagnostic performance of the DL models with that of a radiologist; and third, to investigate the benefits of transfer learning that involves evaluating whether a small amount of data derived from the target domain would improve the performance of DL models across different MRI vendors.

\section{Materials And Methods}

Istanbul Mehmet Akif Ersoy Research and Training Hospital Ethics committee approved this retrospective multicenter study (Approval number: 2019-77) and waived the need for informed consent. All procedures performed in studies involving human participants were in accordance with the ethical standards of the institutional and/or national research committee and with the 1964 Helsinki declaration and its later amendments or comparable ethical standards. The dataset was obtained from six tertiary care centers. Six medical doctors reviewed the radiology reports of consecutive brain DWIs of adult patients obtained with clinical suspicion of acute ischemic stroke between January 2012 and October 2019 using several keywords (e.g., stroke, ischemia, limb weakness, and diffusion-restriction). The exclusion criteria were as follows: (1) DWI obtained 24 hours after the onset of the symptoms; (2) patients with a primary brain tumor, metastatic brain tumors, or demyelinating lesions; (3) DWI with severe motion or metallic artifacts; and (4) incomplete imaging or clinical data (i.e., no information on the ischemia time or lack of high bvalue images or apparent diffusion coefficient maps). Detailed information regarding the patient selection process is depicted in Figure 1.

Of the six study centers, three had 1.5T Genera Electronics MRI scanners (Optima MR450w, Signa HDxt, and Signa Explorer; GE Healthcare, Milwaukee, WI, USA) and the other three had 1.5T Siemens scanners (Aera, Avanto, and Symphony, Siemens Healthineers, Erlangen, Germany). The data of the six centers were divided into two parts based on the MR scanner; dataset A included DWIs obtained using a Siemens scanner, and dataset B included DWIs obtained with a GE scanner. Detailed information regarding the DWI protocols at each center is provided in Supplementary Table S1.

\section{Ground-Truth Segmentations}

Six neuroradiologists (E.K., O.K., H.H.S., B.K., S.N.D., M.V.) with over 10 years of neuroradiology experience from each center examined the recruited images. The neuroradiologists were free to assess all the available clinical and radiological data during the evaluation. Briefly, the neuroradiologist evaluated the images for acute ischemia; acute ischemic lesions were defined as those with hyperintense signal on diffusion-weighted (DW) images with high b-values and corresponding hypointensities on apparent 
diffusion coefficient (ADC) maps [22]. If a neuroradiologist decided that a scan had undiagnostic image quality or had no visible acute ischemic lesion, then the patient was excluded from the study. Subsequently, DW images with the highest b-values and corresponding ADC maps of the patients were anonymized. A unique identification number was assigned to each patient for further analyses.

Anonymized $A D C$ maps and DWls were imported into a known open-source software for segmentation (ImageJ, https://imagej.nih.gov). The neuroradiologists performed segmentations on the DW images using a free-hand region of interest. The segmentation quality of the test sets of datasets $A$ and $B$ was mandatory to achieve reliable performance comparisons. The neuroradiologists re-drew the segmentations on the same images of the test sets in two different sessions after an interval of 1 month. To this end, each patient in the test sample had three different segmentation masks provided by the same neuroradiologists. A intra-reader majority voting was used to create ground-truth masks of the test sets. The pixels accounted as positive for an ischemic lesion in two or more masks were accepted as positive, and those accounted as negative for an ischemic lesion in two or more masks were accepted as negative.

\section{Models}

We employed a well-known CNN architecture for biomedical image segmentation, U-net, or U-shaped networks, but made several modifications [23]. The original U-net model has two main components: the encoder, which serves to identify the most representative features of the images, and the decoder, in which the up-sampling process is performed to regain spatial resolution while preserving the highrepresentative power of the feature maps for precise segmentation. The concatenations between the encoder and decoder facilitate the network's ability to preserve the spatial information of the pixels. U-net can work on both 2D and three-dimensional (3D) data [23, 24]. Hypothetically, using 3D U-net might appear to be the best option since DWls consist of a stack of slices and these images are routinely interpretated in a three-dimensional fashion by a radiologist. However, 3D U-net requires higher memory capacity and it might require lowering the original spatial resolution or using patch-based approaches in the network, which inevitably leads to loss if contextual information $[25,26]$. In contrast, 2D networks suffer from a lack of 3D, or sequential, interpretability of their 3D counterparts; therefore, they might lead to inferior performance [14].

To mitigate the drawbacks of 3D U-net while preserving spatial contextual information, we used a residual ConvLSTM U-Net, which is a hybrid network architecture that leverages the high spatial and sequential representational capacity of convolutional and recurrent neural networks as well as exploits the skip connections that facilitate information flow throughout the network [27, 28]. Figure 2 illustrates the details of the proposed residual ConvLSTM U-Net architecture.

\section{Experiments}

The stack of high b-value DW images and corresponding ADC maps were fed into the network on a patient-basis using two different channels. Following typical image pre-processing operations were performed on the images before feeding them into the network: (1) intensity normalization within $0-1$; (2) resampling the images into $224 * 224$ pixels; (3) and image windowing, which is determined as the best 
window level for the neuroradiologist's eye for assessing DWls for each center and scanner. Several data augmentations, cropping, rotation, flipping, and elastic deformations were implemented on the go.

All DL experiments were conducted using a high-level DL library, Keras on TensorFlow (Tensorflow 1.4 Google LLC, Mountain View, CA). The total trainable parameters of the residual ConvLSTM U-Net were $8,228,401$. The hyperparameters of the models were optimized using the validation partition and were as follows: loss function was Tversky loss (alfa $=0.5$, beta $=0.5$ ); number of epochs was 100 ; optimizer was Adam; and learning rate was $1 \mathrm{e}-5$. The total training time for models $A$ and $B$ was 10.5 and $12 \mathrm{~h}$, respectively.

Datasets A and B were split into three parts as the training (80\%), validation (10\%), and internal test (10\%) sets. The best model was selected based on its performance on the validation data. The DL models trained on datasets $A$ and $B$ were referred as models $A$ and $B$. The segmentation performance of models $A$ and $B$ was first assessed on the internal test sets consisting of images from the same manufacturer. Subsequently, their performances were evaluated on the test partition of the other dataset (i.e., model $A$ on the test set of dataset $B$ and vice versa), and these assessments were referred to as external tests.

Furthermore, to simulate a scenario of extensive available imaging data from one manufacturer while it is limited from another, we utilized transfer learning [29]. The validation parts of each dataset were used to fine-tune the pre-trained model on the other dataset (e.g., pre-trained model A was fine-tuned with the validation part of dataset $B$ and vice versa) for approximately 20 epochs with a learning rate of $1 \mathrm{e}-6$. These models were referred as fine-tuned models $A$ and $B$, respectively. Figure 3 shows the DL experiment pipeline of the present work.

\section{Evaluating the Performances}

The primary metric for investigating a model's performance was the Dice coefficient (two * areas of overlap/total pixels combined), which is a measure of overlap between the model's predictions and the ground truth [30]. The Dice coefficient ranges between 0 and 1, where 1 represents a complete match between the ground truth and prediction, while 0 reflects no match. To compare the performance of the DL models with that of a radiologist, another radiologist with 8 years of experience (D.A.) manually delineated the borders of the ischemic lesions on DWI on the test partitions.

\section{Statistical analysis}

Statistical analysis was performed using Scipy library v1.5.4 of Python programming language ("https://docs.scipy.org"). Categorical variables are presented as frequencies and percentages. Continuous variables were investigated using distribution plots and the Shapiro-Wilk test to assess for normality. Normally distributed continuous variables are presented as mean, standard deviations, and ranges, while non-normally distributed continuous variables are presented as median and interquartile ranges. Mann-Whitney $U$ test was used to compare each model's performance on its internal and external tests. Wilcoxon test was used to compare the performances of fine-tuned and native models $\mathrm{A}$ 
and $B$ on the external test sets. Mann-Whitney $U$ test was used to compare the Dice scores of the models and the radiologist on the test sets. A p-value $<5 \%$ was considered as a statistically significant result.

\section{Results}

Dataset A consisted of 2986 patients (1793/2986 men, $60 \%)$ with a mean age of $65.80 \pm 11.28$ years (range, 47-85), and dataset B comprised 3951 patients (2223/3951 men, 56.26\%) with a mean age of $63.07 \pm 10.78$ years (range, $45-81$ ). The training, validation, and internal test samples of datasets $A$ and $B$ included 2385, 301, and 300 images and 3157, 397, and 397 images, respectively. The median time interval between the onset of symptoms and imaging was 6.5 hours (interquartile range, IQR, 4.5-8) and 7 hours (IQR, 6-8) in datasets A and B, respectively. Detailed information on the study sample is provided in Table 1. 
Table 1

The detailed characteristics of the study sample.

\begin{tabular}{|c|c|c|}
\hline Variables & Dataset A $(n=2986)$ & Dataset B $(n=3951)$ \\
\hline \multicolumn{3}{|l|}{ Age (years) } \\
\hline Total dataset & $65.80 \pm 11.28(47-85)$ & $63.07 \pm 10.78(45-81)$ \\
\hline Training set & $65.81 \pm 11.23(47-85)$ & $63.01 \pm 10.78(47-85)$ \\
\hline Validation set & $67.09 \pm 11.29(48-84)$ & $63.83 \pm 10.98(45-72)$ \\
\hline Test set & $65.26 \pm 11.64(51-81)$ & $62.76 \pm 10.52(45-71)$ \\
\hline \multicolumn{3}{|c|}{ Male gender (\%) } \\
\hline Total dataset & $1793 / 2986(60 \%)$ & $2223 / 3951$ (56.26\%) \\
\hline Training set & $1484 / 2385(62.22 \%)$ & 1833/3157 (58.06\%) \\
\hline Validation set & 159 / $301(52.82 \%)$ & 204/397 (51.38\%) \\
\hline Test set & $150 / 300(50 \%)$ & 197/397 (49.62\%) \\
\hline \multicolumn{3}{|c|}{ Symptoms to MRI (hours) } \\
\hline Total Dataset & $6.5(4.5-8)$ & $7(6-9)$ \\
\hline Training set & $6(4.5-8)$ & $7(6-9)$ \\
\hline Validation set & $5.5(4.5-7.5)$ & $6.5(5-10)$ \\
\hline Test set & $7.5(6-10)$ & $8(3-11)$ \\
\hline \multicolumn{3}{|c|}{ Manual lesion Volumes (mL) } \\
\hline Total Dataset & $3.66(2.51-15.55)$ & $3.25(2.62-6.81)$ \\
\hline Training set & $3.95(2.61-17.44)$ & $3.27(2.63-6.94)$ \\
\hline Validation set & $3.41(2.43-15.04)$ & $3.21(2.58-6.25)$ \\
\hline Test set & $2.64(2.19-4.68)$ & $3.18(2.55-6.42)$ \\
\hline
\end{tabular}

The median Dice scores of model A were 0.866 (IQR, 0.774-0.918), 0.835 (IQR, 0.702-0.899), and 0.858 (IQR, 0.752-0.909) in the training, validation, and internal test tests, respectively. The median Dice scores of model B were 0.896 (IQR, 0.813-0.940), 0.865 (IQR, 0.745-0.923), and 0.857 (IQR, 0.723-0.921) in the training, validation, and internal test tests, respectively.

Model A yielded a median Dice score of 0.734 (IQR, 0.56-0.843) in the external test consisting of images from dataset $B$. There was a significant difference between model A's performance on the internal and external test sets in Dice scores (0.866 vs. 0.734 , respectively, $P<0.0001)$. Model $B$ yielded a median Dice score of 0.756 (IQR, $0.613-0.851)$ in the external test consisting of images from dataset A. There was a 
significant difference between model B's performance on the internal and external test sets in Dice scores (0.857 vs. 0.756 , respectively, $P<0.0001)$.

\section{Radiologist versus DL}

The radiologist achieved a median Dice score of 0.868 (IQR, 0.762-0.895) and 0.867 (IQR, 0.733-0.899) on the test partitions of datasets $A$ and $B$, respectively. Model A's performance, compared with that of the radiologist, on dataset A's internal test was not significantly different $(0.868$ vs. 0.858 , respectively, $P=$ 0.273). Similarly, model B's performance, compared with that of the radiologist, on dataset B's internal test was not significantly different $(0.867$ vs. 0.857 , respectively, $P=0.668)$. Furthermore, the models' performances on the external test sets were compared with those of the radiologist. The Mann-Whitney U model identified that models $A$ and $B$ demonstrated significantly lower performance than that of the radiologist on the external tests. Model A yielded a median Dice score of 0.734 (IQR, 0.56-0.843), while the radiologist achieved a median Dice score of 0.867 (IQR, 0.733-0.899) on the test partition of dataset $B(P<0.0001)$. Model $B$ yielded a median Dice score of 0.756 (IQR, 0.613-0.851), while the radiologist achieved a median Dice score of 0.868 (IQR, 0.762-0.895) on dataset A's test partition $(P<0.0001)$.

\section{DL with Transfer Learning}

Fine-tuned model A achieved a median Dice score of 0.832 (IQR, 0.671-0.916) when applied to the external test consisting of images from dataset $B$. Wilcoxon test revealed that fine-tuned model $A$ achieved statistically higher performance than native model $A$ on the external test ( 0.818 vs. 0.734 , respectively, $\mathrm{P}<0.0001)$. Additionally, fine-tuned model $A$ demonstrated non-inferior performance compared with that of the radiologist $(0.832$ vs. 0.867 , respectively, $P=0.127)$. Fine-tuned model $B$ achieved a median Dice score of 0.846 (IQR, 0.730-0.902) when applied to the external test consisting of images from dataset $A$. Fine-tuned model $B$ yielded a higher performance compared with native model $B$ on the external test $(0.846$ vs. 0.756 , respectively, $P<0.0001)$. Similarly, fine-tuned model $A$ demonstrated non-inferior performance compared with that of the neuroradiologist $(0.846 \mathrm{vs} .0 .868$, respectively, $\mathrm{P}=$ 0.468). Figures 4 illustrates the segmentation of the DL model.

\section{Discussions}

In the present work, a residual ConvLSTM U-Net was applied for segmentation of acute ischemic lesions on DWI using a large-scale multicenter dataset to investigate the performance of the DL models across different MRI manufacturers. This study makes several contributions to the existing literature on DLbased acute ischemic lesion segmentation on DWI. (1) The DL models demonstrated non-inferior performance compared with a radiologist in delineating borders of ischemic lesions when applied to previously unseen images derived from the same manufacturer on which the models were previously trained. (2) The DL models yielded reasonable performance when applied to DW images derived from a different MRI manufacturer with a median Dice score of 0.734-0.756. (3) However, the segmentation performance of the DL models was substantially higher when applied to previously unseen DW images of the same manufacturer compared with that of DL models applied to previously unseen images of a 
different manufacturer. (4) In contrast, the radiologist's performance did not differ between datasets derived from different MRI manufacturers. (5) When the DL models were fine-tuned with a small fraction of the images obtained from the other manufacturer using transfer learning, their performance increased substantially to a non-inferior level to that of a radiologist on the external tests.

Chen et al. [19] were some of the first to demonstrate the potential of DL algorithms in ischemic lesion segmentation on DWI on a large scale. The authors proposed two sequential CNNs, an ensemble of DeconvNets followed by a multi-scale CNN. The authors reported a mean Dice coefficient of 0.67 in 741 participants. However, all their images were derived from an MRI scanner of the same manufacturer. Kim et al. [16] used a 2D U-net to segment acute ischemic lesions on DWI with corresponding ADC maps in 296 participants and compared its performance with a commercially available stroke imaging software. The authors demonstrated that their DL model achieved similar performance to that of the commercially available software. However, the study was limited by the small sample size and the domination of DWIs from a single vendor. Zhang et al. [14] investigated the performance of deep 3D fully convolutional DenseNets for ischemic lesion segmentation on DWI and ADC in 242 patients. The authors were obligated to resample the original spatial resolution of the images to a lower dimension to use a deep 3D model due to memory constraints, but achieved excellent performance with a mean Dice similarity coefficient of 0.79 . Their dataset was derived from MRI scanners of two vendors; however, the authors did not mention the distribution of the images regarding the manufacturer.

In a large-scale multicenter international study by Wu et al. [18], an ensemble 3D CNN, DeepMedic framework, was used to segment acute ischemic lesions on DW with their ADC maps in a heterogeneous cohort. Our study shared similar foundations with their work because they also tried to demonstrate the applicability of DL in a diverse patient population to reveal its usefulness in practice. The authors included 2770 patients but the ground-truth segmentations were only available for approximately a quarter of the data. The authors achieved a mean Dice score of 0.77 and highlighted the benefits of using the ensemble methods. Furthermore, they provided essential insights into the use of DL for acute ischemic lesion segmentation on DWI, such as the negative effects of different b-values on a model's segmentation performance and the robustness of DL models across different field strengths.

We acknowledge that the heterogeneous nature of the acute ischemic lesions, in addition to the technical variations and differences in the DL architectures used to evaluate the lesions, inevitably hampers the comparability of studies on DL-based ischemic lesion segmentation. In recent years, there have been some attempts, such as the ischemic lesion segmentation challenge, to create publicly available datasets for investigating the performance of DL models [31]. However, the existing datasets were of small sizes and lacked variability regarding MRI scanner vendors [31]. Therefore, we suggest that it is not possible to compare the performance of the proposed DL-based solutions for acute ischemic lesion segmentation.

Nevertheless, we did not aim to compare the performances of our DL models with those proposed in the earlier works but rather aimed to assess the inter-vendor operability of these models. This is of clinical importance since it is not an unlikely clinical or academic scenario in which a trained DL model should be 
directly used at a center in which the MRI scans are routinely obtained using a scanner from a different manufacturer. As expected, a radiologist did not demonstrate substantial impairment in terms of segmentation performance across different vendors. In contrast, the performance of the DL models was impaired when applied to DW images obtained using a different vendor's scanner.

To this end, we used transfer learning to improve the model's performance across different MR vendors. The DL models that were fine-tuned using only a small part of the dataset from different vendors demonstrated substantial performance improvements. Therefore, the fine-tuned models demonstrated non-inferior performance in acute ischemic lesion segmentation compared with that of an expert radiologist. These findings imply that a base DL model could be readily available across different MRI scanners of manufacturers if it is provided with a relatively small amount of data from the target domain. However, we admit that transfer learning might be beneficial only to some degree in such scenarios because it also requires some labeled images from the target domain. To this end, several unsupervised solutions have been proposed in the context of domain adaptation, which remains an active research area [33].

Several limitations to this study should be acknowledged. Our dataset consisted of only two MRI vendors; therefore, the inter-operability of the DL models across other vendors could not be evaluated. Second, the exclusion criteria (e.g., DW images with severe motion artifacts or tumors) of the present work might result in a bias toward the DL models such that an experienced radiologist might not misdiagnose and delineate the borders of a brain tumor on DW. In contrast, the DL model might not be as accurate as the expert since it was trained for a narrower task. Therefore, DL models might provide inferior performance to an experienced radiologist even when applied to the same MR vendor's images. Third, the present work dataset did not include any DW images obtained with a 3T MR scanner; therefore, we could not compare the applicability of these models across different field strengths.

In conclusion, the DL model, residual ConvLSTM U-Net, demonstrated non-inferior performance to an expert radiologist in segmenting acute ischemic lesions on DWI when applied to previously unseen images derived from the same manufacturer on which the models were previously trained, but its yields worsened across different manufacturers. Notably, fine-tuning the model using a small sample of the images from the different manufacturer (i.e., target domain) substantially increased its performance to a non-inferior level compared with an expert radiologist.

\section{Declarations}

\section{Conflict of interest statement}

All authors declare no conflict of interest.

\section{Ethical Statement and consent to participate}


All procedures performed in studies involving human participants were in accordance with the ethical standards of the institutional and/or national research committee and with the 1964 Helsinki declaration and its later amendments or comparable ethical standards.

\section{Availability of data and material}

Fine-tuned models and several examples of test images are provided in the http://52.59.225.200:8080. The researchers could upload their anonymized high b-value diffusion-weighted images as zip files to the website to test models' performance on their own images. Further data access requests by qualified researchers trained in human subject confidentiality protocols should be sent to the corresponding author.

\section{Funding}

No funding was received.

\section{Author Contributions}

D.A.- Writing the draft, conceptualization, statistical analysis, Interpretation of data; M.Y.- Statistical analysis, deep learning experiments; C.A.- Investigation, data curation; C.T.- Investigation, data curation;O.A.- Investigation, data curation; O.B.-data curation; Y.D.S..- data curation; A.U.-data curation; V.S.-data curation; S.N.D- methodology, validation; M.V. - methodology, validation; H.H.S - methodology, validation, study design; B.K. - methodology, validation, study design; I.O.- methodology, critical review of the work, supervision, editing, deep learning experiments; O.K.- methodology, critical review of the work, supervision, editing, conceptualization; E.K.- methodology, critical review of the work, supervision, editing, conceptualization

\section{References}

1. Powers, W.J., et al. Guidelines for the Early Management of Patients With Acute Ischemic Stroke: 2019 Update to the 2018 Guidelines for the Early Management of Acute Ischemic Stroke: A Guideline for Healthcare Professionals From the American Heart Association/American Stroke Association. Stroke. 12, 344-418 (2019). Erratum in: Stroke. 50, 440-441 (2019).

2. Zerna, C., Thomalla, G., Campbell BCV, Rha JH, Hill MD. Current practice and future directions in the diagnosis and acute treatment of ischaemic stroke. Lancet. 392, 1247 (2018).

3. Schellinger, P.D., et al. Therapeutics and Technology Assessment Subcommittee of the American Academy of Neurology. Evidence-based guideline: The role of diffusion and perfusion MRI for the diagnosis of acute ischemic stroke: report of the Therapeutics and Technology Assessment Subcommittee of the American Academy of Neurology. Neurology. 75, 177 (2010).

4. Kuhl, C.K., et al. Acute and subacute ischemic stroke at high-field-strength (3.0-T) diffusion-weighted MR imaging: intraindividual comparative study. Radiology. 234, 509 (2005). 
5. Zaidi, S.F., et al. Final infarct volume is a stronger predictor of outcome than recanalization in patients with proximal middle cerebral artery occlusion treated with endovascular therapy. Stroke. 43, 3238-3244 (2012).

6. Lansberg, M.G., et al. MRI profile and response to endovascular reperfusion after stroke (DEFUSE 2): a prospective cohort study. Lancet Neurol. 11, 860-867 (2012).

7. Yoo, A.J., et al. MRI-based selection for intra-arterial stroke therapy: value of pretreatment diffusionweighted imaging lesion volume in selecting patients with acute stroke who will benefit from early recanalization. Stroke. 40, 2046-2054 (2009).

8. Sanák, D., et al. Impact of diffusion-weighted MRI-measured initial cerebral infarction volume on clinical outcome in acute stroke patients with middle cerebral artery occlusiontreated by thrombolysis. Neuroradiology. 48, 632-639 (2006).

9. Prakash, K.N.B., Gupta, V., Bilello, M., Beauchamp, N.J., Nowinski, W.L. Identification, segmentation, and image property study of acute infarcts in diffusion-weighted images by using a probabilistic neural network and adaptive Gaussian mixture model. Acad Radiol. 13, 1474-1484 (2006).

10. Purushotham, A., et al. Apparent diffusion coefficient threshold for delineation of ischemic core. Int $J$ Stroke. 10, 348-353 (2015).

11. LeCun, Y., Bengio, Y., Hinton, G. Deep learning. Nature. 521, 436-444 (2015).

12. Lakhani, P., Gray, D.L., Pett, C.R., Nagy, P., Shih, G. Hello World Deep Learning in Medical Imaging. J Digit Imaging. 31, 283-289 (2018).

13. Kumar, A., et al. CSNet: A new DeepNet framework for ischemic stroke lesion segmentation. Comput Methods Programs Biomed. 193, 105524 (2020).

14. Zhang, R., et al. Automatic Segmentation of Acute Ischemic Stroke From DWI Using 3-D Fully Convolutional DenseNets. IEEE Trans Med Imaging. 37, 2149-2160 (2018).

15. Woo, I., et al. Fully Automatic Segmentation of Acute Ischemic Lesions on Diffusion-Weighted Imaging Using Convolutional Neural Networks: Comparison with Conventional Algorithms. Korean J Radiol. 20, 1275-1284 (2019).

16. Kim, Y.C., et al. Evaluation of Diffusion Lesion Volume Measurements in Acute Ischemic Stroke Using Encoder-Decoder Convolutional Network. Stroke. 50, 1444-1451 (2019).

17. Winzeck, S., et al. Ensemble of Convolutional Neural Networks Improves Automated Segmentation of Acute Ischemic Lesions Using Multiparametric Diffusion-Weighted MRI. AJNR Am J Neuroradiol. 40, 938-945 (2019).

18. Wu, O., et al. Big Data Approaches to Phenotyping Acute Ischemic Stroke Using Automated Lesion Segmentation of Multi-Center Magnetic Resonance Imaging Data. Stroke. 50, 1734-1741 (2019).

19. Chen, L., Bentley, P., Rueckert, D. Fully automatic acute ischemic lesion segmentation in DWI using convolutional neural networks. Neuroimage Clin. 13, 633-643 (2017).

20. Pooch, E.H.P., Ballester, P.L., Barros, R.C. Can we trust deep learning models diagnosis? The impact of domain shift in chest radiograph classification. Preprint at https://arxiv.org/abs/1909.01940 (2019). 
21. Lampert, T., et al. Strategies for Training Stain Invariant CNNS. 2019 IEEE 16th International Symposium on Biomedical Imaging. Venice, Italy, 2019: 905-909. https://ieeexplore.ieee.org/abstract/document/8759266. Accessed October 20, 2020.

22. Srinivasan, A., Goyal, M., Al Azri, F., Lum, C. State-of-the-art imaging of acute stroke. Radiographics. 26, 75-95 (2006).

23. Ronneberger, O., et al. U-Net: Convolutional Networks for Biomedical Image Segmentation. In: Navab, N., Hornegger, J., Wells, W., Frangi, A. (eds) Medical Image Computing and Computer-Assisted Intervention - MICCAI 2015. MICCAI 2015. Lecture Notes in Computer Science, vol 9351. (Springer, Cham., 2015).

24. Çiçek, Ö., et al. 3D U-Net: Learning Dense Volumetric Segmentation from Sparse Annotation. In: Ourselin, S., Joskowicz, L., Sabuncu, M., Unal, G., Wells, W. (eds) Medical Image Computing and Computer-Assisted Intervention - MICCAI 2016. MICCAI 2016. Lecture Notes in Computer Science, vol 9901. (Springer, Cham., 2016).

25. Payan, A., Montana, G. Predicting Alzheimer's disease: a neuroimaging study with 3D convolutional neural networks. Preprint at https://arxiv.org/abs/1502.02506 (2015).

26. Nemoto, T., et al. Efficacy evaluation of 2D, 3D U-Net semantic segmentation and atlas-based segmentation of normal lungs excluding the trachea and main bronchi. J Radiat Res. 23, 257-264 (2020).

27. Zhang, Z., Liu, Q., Wang, Y. Road extraction by deep residual u-net. IEEE Geoscience and Remote Sensing Letters. 15, 749-753 (2018).

28. Shi, X., et al. Convolutional LSTM network: A machine learning approach for precipitation nowcasting. Advances in neural information processing systems. 28, 802-810 (2015).

29. Pan, S.J., Yan, Q. A survey on transfer learning. IEEE Trans Knowl Data Eng. 22, 1345-1359 (2010).

30. Dice, L.R. Measures of the Amount of Ecologic Association Between Species. Ecology. 26, 297-302 (1945).

31. Maier, O., et al. ISLES 2015- A public evaluation benchmark for ischemic stroke lesion segmentation from multispectral MRI. Med Image Anal. 35, 250-269 (2017).

32. Oktay, O. et al. Multi-input Cardiac Image Super-Resolution Using Convolutional Neural Networks. Ourselin, S., Joskowicz, L., Sabuncu, M., Unal, G., Wells, W. (eds) Medical Image Computing and Computer-Assisted Intervention- MICCAI 2016. MICCAI 2016. Lecture Notes in Computer Science, vol 9902. (Springer, Cham. , 2016).

33. Levgen, R., et al. Advances in Domain Adaptation Theory. (ISTE Press- Elsevier, 2019).

\section{Figures}


Diffusion-weighted examinations obtained due to suspicion of stroke $(n=15837)$

\section{Inclusion criteria}

- Adult patients with acute ischemic stroke

- Having DWI obtained within 24 hours after the onset of the symptoms

\section{Exclusion criteria}

- No ischemic lesion on DWI $(n=7813)$

- Presence of mass lesion ( $n=158)$

- Demyelinating disorders $(n=86)$

- Severe Motion artifacts \& Metal artifacts $(n=102)$

- Incomplete data $(n=741)$

Study Sample $(n=6937)$

Dataset A

- DWI with Siemens MRI scanner $(n=2986)$
Dataset B

- DWI with GE MRI scanner $(n=3951)$

\section{Figure 1}

The patient selection process in the current study.

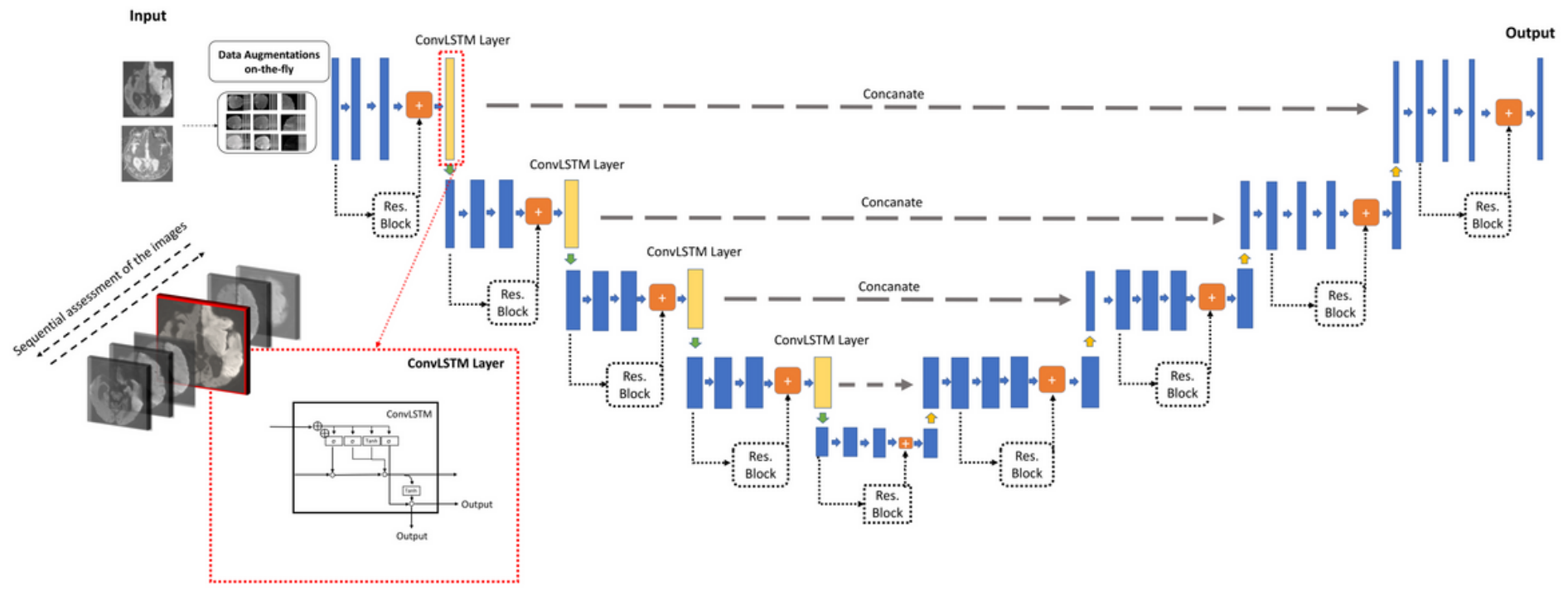

Figure 2 
Residual two-dimensional convolutional long short-term memory (ConvLSTM) U-net. The stack of high bvalue diffusion-weighted images and corresponding apparent diffusion coefficient maps are fed into the network per-patient. Two important modifications are made to the 2D U-net. First, residual layers are utilized for each convolutional block, which allows unimpeded propagation of information throughout the network and mitigates the vanishing gradient problem. Second, bi-directional ConvLSTM layers are implemented on top of each convolutional block of the encoder network to allow communication of the feature maps. Consequently, it enables the network to consider all of the slices of an examination before delineating an ischemic lesion's borders. Therefore, we suggest that this architecture, to some extent, mimics how radiologists assess images, which involves sequential assessment of all slices of an examination before making the final diagnosis or, in this context, performing segmentation.

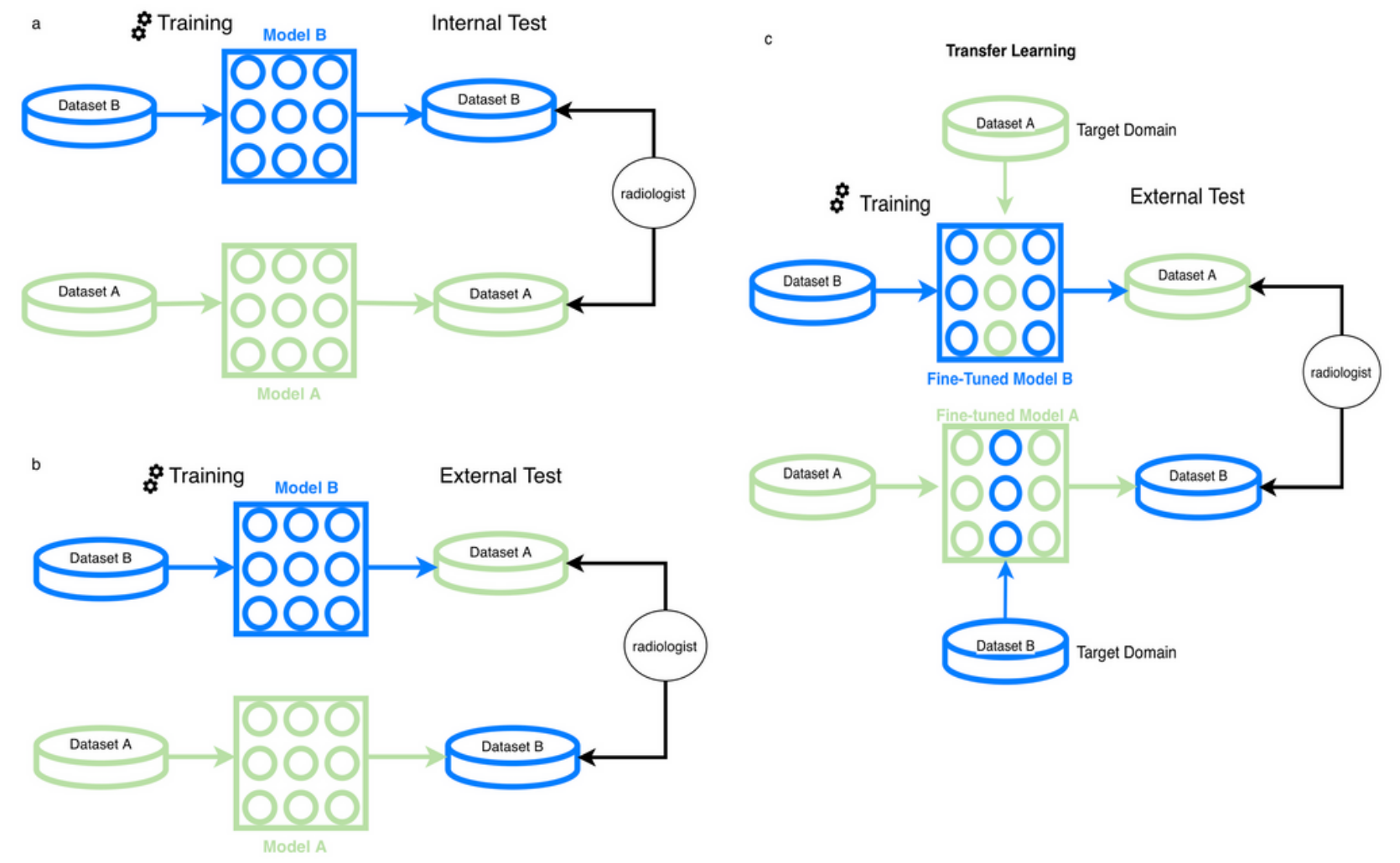

\section{Figure 3}

The flowchart of the deep learning experiments. (A) The deep learning models trained on datasets $A$ and $B$ were referred as models $A$ and $B$. The segmentation performance of models $A$ and $B$ were first assessed on the internal test sets that consisted of images from the scanners of the same manufacturer. (B) Subsequently, the models' performances were evaluated on the test partition of the other dataset, which was referred as external test. (C) The validation parts of each dataset were used to fine-tune the pre-trained model using transfer learning. These models were referred as fine-tuned models $A$ and $B$, respectively. Subsequently, the fine-tuned models' performances were assessed on the external test sets. A single expert radiologist made segmentations on the test partitions of the datasets for performance comparisons with the deep learning models. 

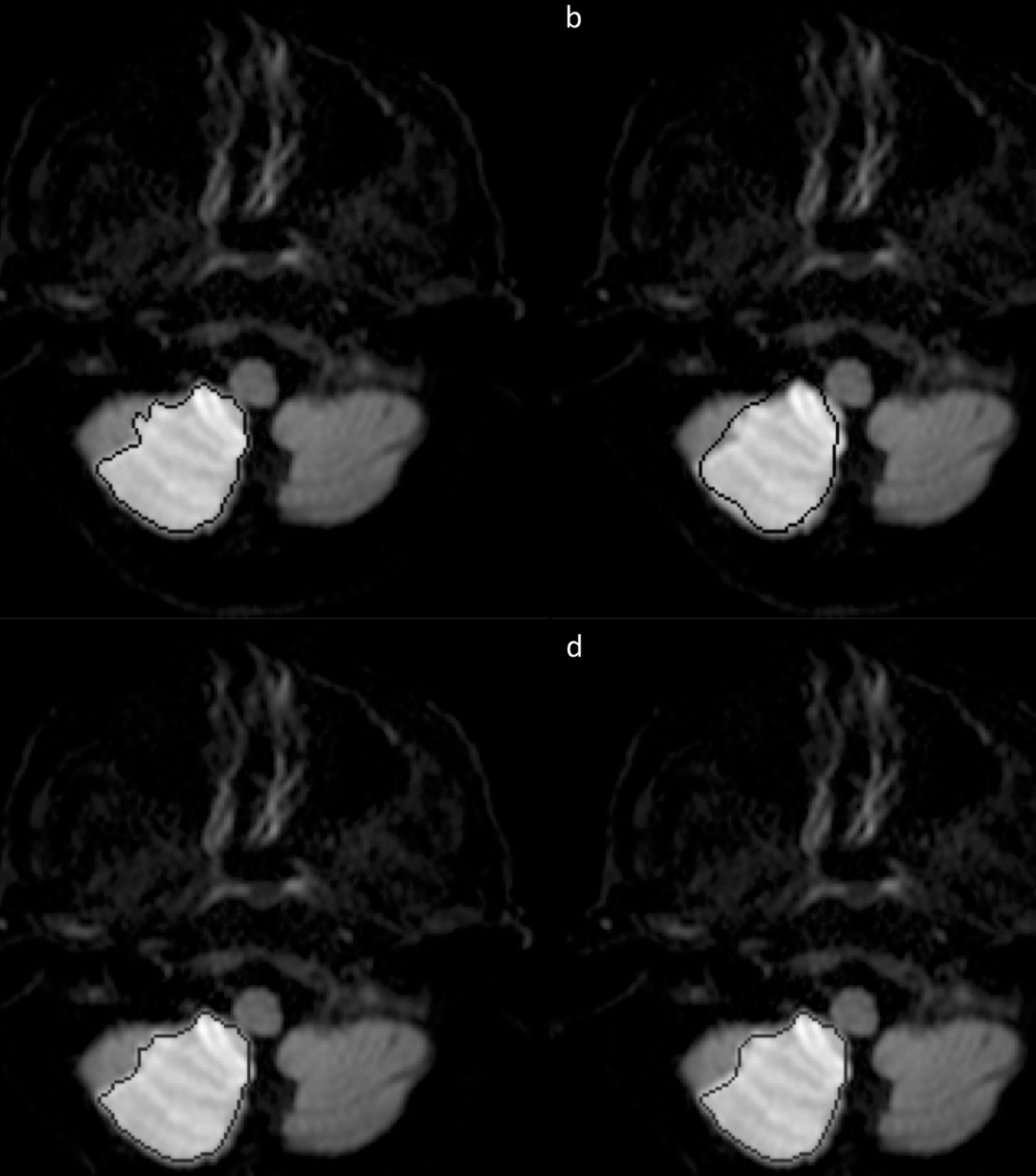

\section{Figure 4}

Acute ischemic lesion segmentation of model B on the external test. Figures shows images of a 64-yearold male patient with acute ischemic lesion in the right cerebellar hemisphere. (A) Ground-truth segmentation mask created by the neuroradiologist. (B) The segmentation of native model B. Note the incorrect contours, which is more profoundly marked in the lateral part of the lesion. (C) The segmentation by the radiologist. (D) The segmentation by fine-tuned model B. Note that the fine-tune 
model demonstrates similar segmentation performance to that of the radiologist, while delineating the borders of the lesion much correctly than the native model.

\section{Supplementary Files}

This is a list of supplementary files associated with this preprint. Click to download.

- Supplementarytable.docx 